Ann. Génét. Sél. anim., 1 (3), 273-280.

\title{
RELATIONS ENTRE LES INDEX LAITIERS DES REPRODUCTEURS MÂLES PÈRES ET FILS DE RACE BOVINE FRANÇAISE FRISONNE PIE-NOIRE
}

\author{
J.-C. MOCQUOT et M. POUTOUS \\ Station de Génétique quantitative et appliquée, \\ Centre national de Recherches zootechniques, 78 - Jouy-en-Josas \\ Institut national de la Recherche agronomique
}

\section{SOMMAIRE}

L'efficacité globale d'une sélection opérée à partir des résultats d'index laitiers a été estimée en comparant les relations observées et théoriques entre les index pour la production de lait et de matière grasse de 186 taureaux F.F.P.N. et les index correspondants de leurs pères.

L'efficacité moyenne est de 91 p. 100 du maximum pour la production de lait et de 78 p. 100 pour celle de matière grasse. Elle augmente avec le coefficient de détermination de l'index. Lorsque celui-ci est supérieur à 0,70 (taureaux testés), on retrouve sur l'index du fils de 40 à 50 p. 100 de l'écart à la moyenne de l'index du père.

Sous réserve de vérification sur un échantillon plus important, il apparait ainsi que la méthode de calcul des index est satisfaisante et que le choix des taureaux pères d'après leur index constitue un élément essentiel de la sélection laitière.

\section{INTRODUCTION}

L'étude des relations entre les index laitiers des reproducteurs mâles pères et fils présente un double intérêt. En premier lieu, elle permet de juger la validité à la fois des hypothèses à la base de la méthode théorique d'estimation des index et des techriques de calcul proprement dites. En effet, si la collecte de l'échantillon de travail est correcte, les erreurs sur les index du père et du fils sont indépendantes. En deuxième lieu, de telles études sont nécessaires pour améliorer et préciser les limites de la sélection sur ascendance, seule capable d'entraîner un progrès génétique à long terme. Ce point revêt une importance particulière au moment où se mettent en place des schémas comportant la mise en testage de reproducteurs fils de mâles favorablement testés. L'étude a été limitée au cas des index lait et matière grasse, l'efficacité d'une sélection sur ascendance pour les taux butyreux et azoté ayant été prouvée à de nombreuses reprises. 
La plus importante étude réalisée sur ce sujet est celle de RoBERTson et ODEGARD (I966) sur I260 couples père-fils de taureaux frisons. Ces auteurs ont estimé à $0,24 \pm 0,02$ la valeur du coefficient de régression de l'index du fils sur celui du père. Par comparaison à la valeur attendue 0,35 , ils en concluent que la méthode de comparaison aux contemporaines de RoBERTSON a une efficacité pratique de 70 p. Ioo. De plus, l'efficacité reste la même quel que soit le nombre de filles des taureaux pères. Sur 86 couples de données, OsingA (I962) trouve une valeur comparable pour le coefficient de corrélation $(r=0,33)$, tandis que des coefficients plus élevés sont obtenus par Vos et Poiritiek (I964) $(r=0,49 \pm 0,07$ pour I ro couples de taureaux frisons, les index étant établis à partir de la comparaison mère-fille), par Politiek (I968) $(r=0,4 \mathrm{I}$ pour 86 couples) et par ENGELER et HERzoG (I965) ( $r=0,39$ pour I3I couples). Pour des béliers de race Lacaune dont les index sont calculés suivant une méthode très voisine de celle employée dans le cas des bovins, Mocouot, Flamant et Poutous (I969) ont trouvé que l'efficacité était fonction du nombre de filles avec une valeur moyenne de $72 \mathrm{p}$. Ioo pour ro filles au moins.

\section{MATÉRIEL ET MÉTHODES}

Le travail a porté sur les index de production de lait et de matière grasse par lactation. L'échantillon de calcul comprenait les 186 couples d'index père-flis de taureaux de race $F . F . P . N$. que nous avons retrouvés dans les résultats de l'ensemble de la race (BoDILIs, 1968) et du C.I.A.* de Charmox. Tous ces mâles étaient connus d'après les résultats d'au-moins 15 filles, un certain nombre avaient été utilisés exclusivement en monte naturelle, d'autres en insémination artificielle, ce dernier groupe comprenant tous ceux testés sur plus de 30 à 35 filles.

Les index avaient été calculés selon la méthode de Poly, Poutous et Frebling (1965), dans laquelle les étables sont réparties en 6 classes d'après la moyenne des lactations de tous âges, les productions, en première lactation uniquement, étant ensuite comparées à la moyenne des contemporaines de même âge et de même classe d'étable. Une correction pour la durée de lactation et le trimestre de vêlage est également effectuée. Le coefficient d'héritabilité retenu étant $h^{2}=$ 0,30 pour les deux caractères, l'index est calculé par la formule :

$$
I=2 \frac{W}{W+13} \frac{\Sigma E}{W}
$$

$\Sigma \mathrm{E}$ étant la somme des écarts, après corrections, entre les filles et leurs contemporaines, W étant la somme des pondérations, obtenue par la formule classique de RoBERTson.

Nous appellerons par la suite coefficient de détermination (CD) de l'index le rapport (2) $\mathrm{CD}=\mathrm{W} /(\mathrm{W}+13)$ dont on peut donner ci-dessous la formule générale en fonction de la variance génétique $\left(\sigma g^{2}\right)$ du caractère et de la variance $\left(s^{2}\right)$ de l'écart moyen $\Sigma \mathrm{E} / \mathrm{W}$ :

$$
\mathrm{CD}=\frac{1}{4} \sigma g^{2} / s^{2}
$$

Nous appellerons efficacité le rapport de la valeur observée sur l'échantillon de travail à la valeur attendue pour les coefficients de corrélation ou de régression. Pour estimer la valeur attendue nous admettrons les hypothèses ci-dessous :

- absence d'épistasie;

- indépendance des actions du génotype et du milieu;

- valeur réelle de l'héritabilité égale à la valeur de travail $\left(h^{2}=0,30\right)$;

- choix des mères indépendant de celui du père (panmixie);

- effectifs de descendantes du père et du fils indépendants.

* Centre d'Insémination artificielle. 
Compte tenu de la formule (3) et du modèle $\mathrm{E}_{n}=\frac{1}{2} g+e_{n}(g:$ valeur génétique du père de variance $\sigma_{g}^{2} ; e_{n}$ : variable aléatoire de variance $\left.\sigma 2\right)$, les valeurs attendues pour la covariance et les coefficients de corrélation et de régression entre l'index du père et celui du fils s'écrivent, en fonction des coefficients de détermination de l'index du père $(\mathrm{C} p)$ et du fils $(\mathrm{C} f)$ :

$$
\begin{aligned}
& \text { Covariance }=\frac{1}{2} \mathrm{C} p \cdot \mathrm{C} f \sigma^{2} ; \\
& \text { Corrélation }=\frac{1}{2} \sqrt{\mathrm{C} p \cdot \mathrm{Cf}} \\
& \text { Régression }=\frac{1}{2} \mathrm{Cf}
\end{aligned}
$$

Pour étudier les résultats en fonction de la précision de l'index, l'échantillon total des données a été subdivisé en 3 sous-échantillons d'après les seuils suivants des coefficients de détermination :

Groupe A : coefficient de détermination compris entre 0,50 et 0,70 pour les 2 éléments du couple (51 couples);

Groupe B : coefficient de détermination compris entre 0,50 et 0,70 pour l'un des éléments du couple et supérieur à 0,70 pour l'autre ( 83 couples);

Groupe C : coefficient de détermination supérieur à 0,70 pour les 2 éléments du couple (52 couples). Ce seuil correspond à $35-40$ filles environ.

Les valeurs des moyennes et des variances et les coefficients de détermination moyens figurent dans le tableau 1; les valeurs de la covariance, des coefficients attendus et observés de régression et de corrélation, de l'efficacité et de l'ordonnée à l'origine de la droite de régression de l'index du fils sur celui du père sont reportés dans le tableau 2.

Dans le tableau 3 se trouvent les valeurs de la covariance et du coefficient de corrélation génétique, calculées selon la méthode de Dickerson (1959), entre les index de lait et de matière grasse.

Enfin, la figure 1 illustre la distribution des couples d'index pour la production de lait dans le cas des mâles du groupe $C(C D>0,70)$. Dans ce groupe, chaque père avait en moyenne 2,4 fils.

\section{TABLEAU I}

\begin{tabular}{|c|c|c|c|c|c|c|}
\hline GROUPE & ORIGINE & $\begin{array}{c}\text { CD } \\
\text { SEUILS }\end{array}$ & $\underset{\text { MOYENS }}{C D}$ & EFFECTIFS & MOYENNES & VARIANCES \\
\hline \multirow{2}{*}{ Total } & père & \multirow{2}{*}{$\geqslant 0,5^{0}$} & $0,8 \mathbf{I}$ & I 86 & $\begin{array}{l}56,40 \\
12,81\end{array}$ & $\begin{array}{l}201465 \\
205,88\end{array}$ \\
\hline & fils & & 0,66 & I 86 & $\begin{array}{l}6,67 \\
5,97\end{array}$ & $\begin{array}{l}200745 \\
310,47\end{array}$ \\
\hline \multirow{2}{*}{$\mathbf{A}$} & père & \multirow{2}{*}{$0,5^{\circ}$ à $0,7^{\circ}$} & 0,60 & $5 \mathrm{I}$ & $\begin{array}{l}48,63 \\
11,43\end{array}$ & $\begin{array}{l}190 \text { I } 30 \\
232,21\end{array}$ \\
\hline & fils & & 0,56 & $5^{I}$ & $\begin{array}{r}32,29 \\
5,14\end{array}$ & $\begin{array}{l}163306 \\
243,20\end{array}$ \\
\hline \multirow{2}{*}{ B } & père & \multirow{2}{*}{$\begin{array}{c}0,50 \text { à } 0,70 \\
\text { pour l'un } \\
>0,70 \\
\text { pour l'autre }\end{array}$} & 0,87 & 83 & $\begin{array}{l}32, \text { I } 2 \\
12,35 \\
\end{array}$ & $\begin{array}{l}207602 \\
196,4^{2}\end{array}$ \\
\hline & fils & & $0,6 \mathbf{I}$ & 83 & $\begin{array}{l}1,52 \\
6,73\end{array}$ & $\begin{array}{l}250657 \\
398,17\end{array}$ \\
\hline \multirow{2}{*}{ C } & père & \multirow{2}{*}{$>0,70$} & 0,93 & $5^{2}$ & $\begin{array}{r}102,77 \\
14,90\end{array}$ & $\begin{array}{l}207223 \\
196,64\end{array}$ \\
\hline & fils & & 0,84 & 52 & $\begin{array}{r}7,96 \\
5,5^{8}\end{array}$ & $\begin{array}{l}\text { 1 } 46725 \\
245,58\end{array}$ \\
\hline
\end{tabular}

Coefficients de détermination (CD) seuils des différents groupes, CD moyens, effectifs, moyennes et variances pour les index des pères et des fils

Les résultats pour les index de lait sont au-dessus de ceux pour les index de matière grasse, valeurs exprimées en kilogrammes. 


\section{RÉSULTATS ET DISCUSSION}

\section{Remarques}

Les résultats de ce travail (tabl. 2) doivent être considérés avec une certaine prudence, étant donné le nombre relativement faible de données dont nous disposions. Par ailleurs, 1 'origine de ces données est variable, seul le groupe $C$ étant formé exclusivement d'index de taureaux utilisés en insémination artificielle. Le groupe $B$, en particulier, n'est pas homogène car il comprend des couples d'index de taureaux utilisés en monte artificielle avec moins de données connues pour les fils $(\mathrm{CD}<$ $0,70)$ que pour les pères $(\mathrm{CD}>0,70)$ mais également des couples de reproducteurs indexés, les pères en monte naturelle $(C D<0,70)$, les fils en insémination artificielle $(C D>0,70)$. Par ailleurs, les génisses dont les productions laitières ont servi au calcul des index ont vêlé à des dates et dans des régions très diverses. II se peut que le niveau génétique de leurs contemporaines ne soit pas resté constant;

\section{TABLEAU 2}

Relations entre les index des pères et des fils pour la production de lait et de matière grasse

Covariances, coefficients de corrélation observés $(\mathbf{r})$ et attendus $(\mathrm{E}(\mathbf{r}))$, efficacité $(\boldsymbol{r} / \mathrm{E}(\mathrm{r}) \times \mathbf{1} 00)$, coefficients de régression de l'index du fils sur celui du père observés (b) et attendus $(E(\mathrm{~b}))$, efficacité $(b / E(\mathrm{~b}) \times \mathrm{roo})$, ordonnées à l'origine (a) de la droite de régression. Productions exprimées en kilogrammes

\begin{tabular}{|c|c|c|c|c|c|c|c|c|c|c|}
\hline CRITÉRE & GROUPE & $\begin{array}{c}\text { EF- } \\
\text { FEC- } \\
\text { TIF }\end{array}$ & $\begin{array}{l}\text { COVA- } \\
\text { RIANCE }\end{array}$ & $r$ & $\mathrm{E}(r)$ & $\begin{array}{c}\text { EF- } \\
\text { FICA- } \\
\text { CI'TÉ } \\
\%\end{array}$ & $b$ & $\mathrm{E}(b)$ & $\begin{array}{c}\text { EF- } \\
\text { FICA- } \\
\text { CITÁ } \\
\%\end{array}$ & $a$ \\
\hline \multirow{4}{*}{ Lait } & Total & I 86 & $6055^{2}$ & $0,298^{* *}$ & 0,366 & $8 \mathrm{I}$ & 0,300 & 0,329 & $9 I$ & $-10,3$ \\
\hline & A & $5 \mathrm{I}$ & $4^{0} 44^{2}$ & 0,228 & 0,289 & 79 & 0,212 & 0,279 & 76 & 22,0 \\
\hline & B & 83 & 54775 & $0,23^{*}$ & 0,363 & 66 & 0,263 & 0,304 & 86 & $-6,9$ \\
\hline & C & $5^{2}$ & 91789 & $0,524^{* *}$ & 0,443 & II 8 & $0,44^{2}$ & 0,420 & 105 & $-53,4$ \\
\hline \multirow{4}{*}{$\begin{array}{c}\text { Matière } \\
\text { Grasse }\end{array}$} & Total & I 86 & $5^{2,75}$ & $0,209^{*}$ & 0,336 & 57 & 0,256 & 0,329 & 78 & 2,79 \\
\hline & A & $5 I$ & 46,32 & 0,195 & 0,289 & 67 & 0,199 & 0,279 & $7 \mathrm{I}$ & 2,86 \\
\hline & B & 83 & 21,77 & 0,077 & 0,363 & $2 \mathrm{I}$ & O, I I I & 0,304 & $3^{6}$ & 5,37 \\
\hline & $\mathrm{C}$ & 52 & III, I9 & $0,506 * *$ & 0,443 & II 4 & 0,565 & 0,420 & I 34 & $-2,85$ \\
\hline
\end{tabular}

* significatif au seuil de $5 \%$,

** significatif au seuil de $1 \%$. 
de ce fait, les moyennes des index pour les pères et pour les fils ne seraient pas comparables. Ceci rend difficile l'interprétation, en valeur absolue, de l'ordonnée à l'origine de la droite de régression.

En contre-partie des raisons de prudence que nous venons d'énumérer, il faut noter une très bonne concordance, pour la production de lait par lactation, entre les résultats de cette étude et ceux d'un travail effectué sur I34 couples d'index père-fils de béliers Lacaune. Bien que les conditions d'élevage des ovins ne soient pas entièrement comparables à celles des bovins (effectif par troupeau très élevé, monte naturelle exclusivement), cet argument important appuie le jugement porté ici sur la méthode de calcul d'index, très voisine pour les deux espèces.

\section{Production laitière}

L'efficacité est plus faible quand on la mesure d'après les coefficients de corrélation que d'après les coefficients de régression (tabl. 2). Ceci s'explique car l'efficacité est le rapport d'une valeur estimée sur l'échantillon à une valeur théorique toujours calculée dans l'hypothèse d'absence de sélection des pères. Or s'il y a eu effectivement sélection, la valeur estimée est restée, en espérance mathématique, stable pour la régression mais a diminué pour la corrélation. Par conséquerrt, l'efficacité de la corrélation a également diminué.

Contrairement aux observations faites par ROBERTSON et ODEGARD (I966), les relations entre les index des pères et des fils semblent plus étroites pour des effectifs de filles élevés. Bien que la signification de l'ordonnée à l'origine de la droite de régression soit, comme nous l'avons remarqué plus haut, difficile à établir, sa variation en sens inverse de l'efficacité atténue, cependant, le sens de la remarque précédente. Malgré tout, il est raisonnable d'admettre une précision pratique des index plus élevée pour les effectifs importants, ne serait-ce qu'à cause d'un nombre d'étables plus fort et, par suite, d'une influence plus faible de ce facteur dont il n'est pas tenu compte dans les calculs actuels. Ce point paraît net dans notre échantillon, le groupe A comprenant des index de mâles utilisés en monte naturelle dont les filles étaient entretenues dans un nombre d'étables restreint. On peut penser également que l'héritabilité réelle pour l'échantillon est inférieure à la valeur que nous avons retenue $\left(h^{2}=0,30\right)$. Si cette hypothèse était exacte, les coefficients de détermination (formules 2 et 3 ) et les valeurs attendues (formule 4) seraient proportionnellement plus surestimés pour les effectifs faibles (groupes A et B) que pour les forts (groupe C). L'efficacité varierait donc bien alors dans le même sens que le nombre de descendants par lot de testage.

A titre d'illustration, la répartition des index des fils en fonction de ceux des pères a été donnée (fig. I) dans le cas des taureaux testés en insémination artificielle (groupe C). Enfin, l'efficacité moyenne pour l'ensemble de l'échantillon (8r \% pour la corrélation et $9 \mathrm{I} \%$ pour la régression), se compare favorablement avec les résultats des auteurs étrangers cités dans l'introduction. De ce fait, la 
méthode de calcul d'index que nous avons employée apparaît comme un assez bon outil de sélection. Elle permettrait en tous cas d'éviter les erreurs flagrantes qu'on remarque sur la figure I (index négatifs pour un père et ses ro fils par exemple). Ces erreurs expliquent la valeur moyenne très faible des index de lait observée pour les pères (tabl. I).

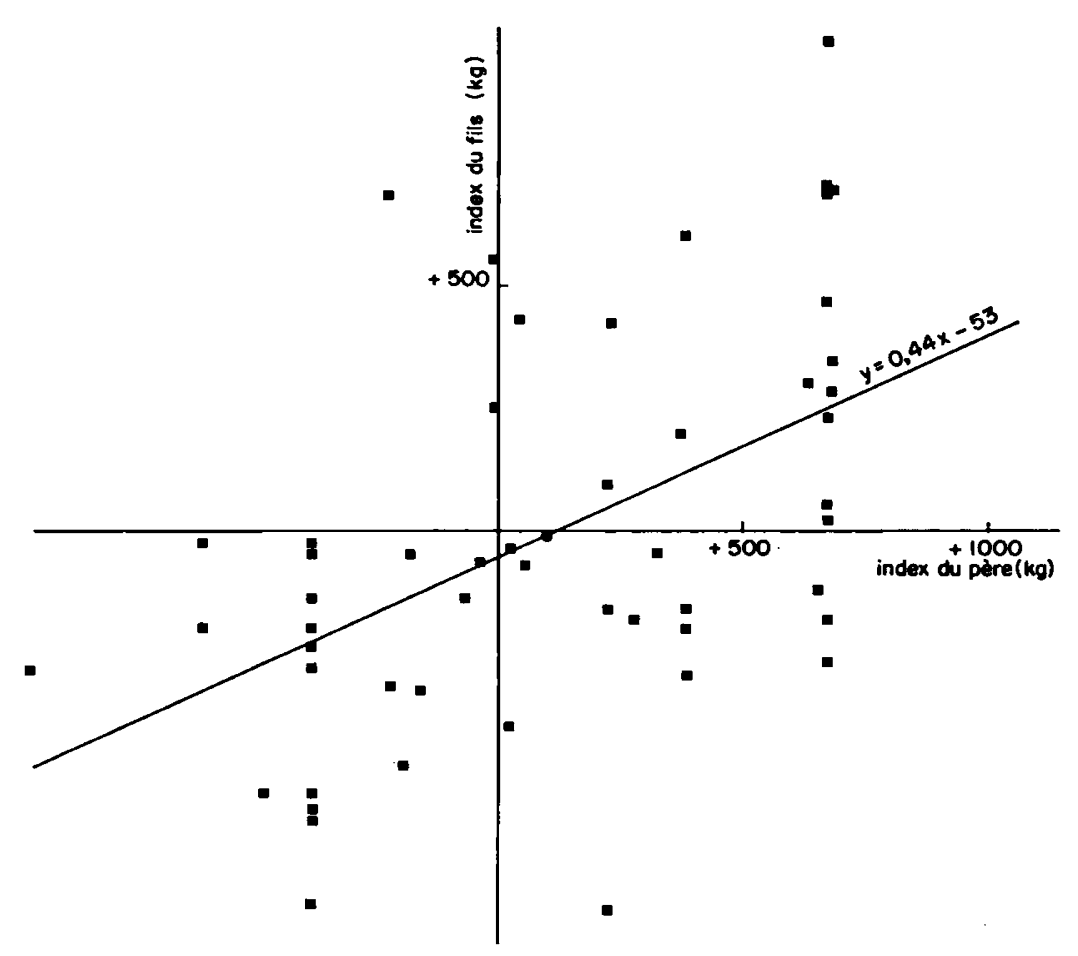

FIG. I. - Répartition des index des fils en fonction de l'index de leur père et droite de régression. Production de lait, groupe $\mathrm{C}(\mathrm{CD}>0,70)$.

\section{Production de matière grasse}

I'étude des relations entre les index des taureaux pères et fils pour la production de matière grasse (tabl. 2) permet d'arriver à des conclusions très voisines de celles obtenues pour la production de lait, sous réserve d'une efficacité moindre et plus fluctuante selon les groupes d'index. Cette différence s'explique, en partie tout au moins, si l'on admet l'existence d'une sélection par la voie femelle et d'un appariement raisonné des parents pour la matière grasse par l'intermédiaire du taux butyreux. Une telle sélection est assez facile, étant donné l'héritabilité élevée du taux butyreux; elle a été effectivement pratiquée dans la race F.F.P.N.. La nature des données et leur faible nombre par sous-lot ne nous permettaient pas de pousser l'analyse plus loin. 
TABLEAU 3

Covariances entre les index des pères (P) et des fils (F) pour la production de lait (L) et de matière grasse (G).

Coefficients de corrélation génétique entre ces deux variables.

\begin{tabular}{|c|c|c|c|c|c|}
\hline \multirow{2}{*}{ GroUPE } & \multicolumn{4}{|c|}{ COVARIANCES } & \multirow{2}{*}{$\begin{array}{l}\text { CORRÉLATTIONS } \\
\text { GÉNÉTIQUES }\end{array}$} \\
\hline & $\mathrm{GF} \times \mathrm{L}, \mathrm{P}$ & $\mathrm{LF} \times \mathrm{GP}$ & $\mathbf{L} \mathbf{F} \times \mathbf{L} \mathbf{P}$ & $\mathrm{GF} \times \mathrm{GP}$ & \\
\hline Total & I 318 & I 284 & 60552 & 52,75 & 0,72 \\
\hline A & 615 & 544 & 40442 & 46,32 & 0,42 \\
\hline B & 565 & 870 & 54775 & 21,77 & 0,66 \\
\hline C & $279 \mathrm{I}$ & 3314 & 9 I 789 & III, 19 & 0,97 \\
\hline
\end{tabular}

\section{Corrélations génétiques}

Les corrélations génétiques entre les index de lait et de matière grasse ont été calculées à partir des couples d'index père-fils (tabl. 3). Elles augmentent considérablement avec le nombre de filles par groupe de testage. La valeur de 0,97 estimée pour les taureaux d'insémination artificielle est très proche de celle obtenue $(0,95)$ dans d'autres conditions pour la même race (Pourous, I964). Il est vraisemblable que plusieurs causes sont à l'origine des faibles valeurs observées pour les groupes A et $\mathrm{B}$. On peut en particulier penser à celles que nous avons avancées précédemment : existence de facteurs de variation non pris en compte dans le calcul des index (nombre d'étables par exemple) et sélection maternelle sur le taux butyreux.

\section{CONCLUSION}

Les objectifs de cette étude étaient limités d'abord par la taille de l'échantillon, ensuite par la méthode suivie qui, comme nous l'avons déjà dit, permet d'estimer l'efficacité globale de l'ensemble des opérations de sélection mais ne met pas en évidence l'origine des erreurs éventuellement constatées. Cependant, de ce travail et de celui fait pour les ovins laitiers, les conclusions suivantes peuvent être provisoirement tirées :

- Les index laitiers tels qu'ils sont établis actuellement fournissent une bonne estimation de la valeur génétique des reproducteurs mâles lorsqu'ils sont établis sur un nombre minimum de performances.

- De ce fait, le choix de jeunes reproducteurs fils de mâles favorablement testés est à conseiller. 
- D'autres études devraient être entreprises pour vérifier ces conclusions et pour améliorer, éventuellement, les méthodes actuelles de calcul des index et les conditions de réalisation pratique du testage.

Rę̧u pour publication en juin 1969.

\section{REMERCIEMENTS}

Ce mémoire a été lu par le Dr R.D. Politiek (Wagermingen) et par M. L. Ollivier (Jouyen-Josas) qui nous ont suggéré d'utiles remarques. Nous voudrions ici témoigner de notre gratitude envers eux.

\section{SUMMARY}

\section{RELATIONSHIPS AMONG THE MILK INDEXES OF SIRE AND SON BREEDING MALES OF THE FRENCH BLACK AND WHITE FRIESIAN BREED}

The efficiency (E) of the method of calculating the index used in France and described by PoLy, Poutous and Frebling (1965; method of intra herd contemporary comparisons) was estimated by comparing the observed and the theoretical relationship of the index of a bull with those of his sons. The coefficients of correlation $(r)$ and of regression $(b)$ were calculated for 186 sire-son pairs of bulls of the French Black and White Friesian breed each of which had at least 15 daughters.

The efficiency increases with the number of daughters tested per bull. It is higher for milk production than for fat production.

Over the total 186 pairs is found :

milk production : $r=0,30, \mathrm{E}=81$ percent; $b=0,30, \mathrm{E}=91$ percent fat production : $r=0,21, \mathrm{E}=57$ percent; $b=0,26, \mathrm{E}=78$ percent

For 52 pairs (males with at least 35 daughters) is found :

milk production : $r=0,52, \mathrm{E}=118$ percent; $b=0.44, \mathrm{E}=105$ percent

fat production : $r=0.53, \mathrm{E}=114$ percent; $b=0.56, \mathrm{E}=134$ percent.

The milk index therefore gives a good idea of the worth of a sire, especially if it is based on at least 40 lactations.

\section{RÉFÉRENCES BIBLIOGRAPHIQUES}

BoDIlıs J., 1968. Étude des principales lignées F.F.P.N., (non publié).

Dickerson G.E., 1959. In "Techniques and procedures in animal production research ", 86-87. Amer. Soc. Anim. Prod., Beltsville.

ENgeler W., Herzog H., I965. Beziehungen zwischen leistungsgeprüften Vätern und ihren Söhnen. Züchtungskunde, 37, I50-I 53 .

Mocquot J.-C., Flamant J.-C., Poutous M., 1969. Aspects quantitatifs de la production laitière de brebis. VI. Relations entre les index laitiers sur ascendance et sur descendance des reproducteurs mâles de race Lacaune. Ann. Génét. Sél. anim., 1,

OSINGA A., 1962. Estimation of the breeding value of bulls on the basis of the production of their ancestors. Vett. Zuivelbericht., 5, 253-260.

Politiek R.D., r968. Prospects of increasing the production of milk protein by breeding. Ned. Melken Zuidveltijdschr., 22, 179-19x.

Poly J., Poutous M., Frebling J., 1965. Méthode de calcul des index de production laitière. Bull. tech. Inform. Ingrs. Serv. A gric., 205, 957-964.

Poutous M., 1964. Le testage des taureaux sur la production en matière azotée de leurs filles. Résultats préliminaires. Ann. Biol. anim. Biochim. Biophys., 4, 273-283.

ROBERTSON A., ODEGARD A.K., I966. A comparison of progeny tests for milk production on fathers and sons. Acta. Agric. Scand., 17, 24 I-246.

Vos P.M., Politrek R.D., I964. Estimation of the breeding value of a young bull on the production figures of his parents. Z. Tierzücht. ZüchtBiol., 79, 3I0-317. 